\title{
Der deutschen Unternehmensmitbestimmung entzogen: Die Zahl der Unternehmen mit ausländischer Rechtsform wächst
}

In der letzten Wirtschaftskrise hat das deutsche Modell der Mitbestimmung mit Aufsichtsrat und Betriebsrat einmal mehr seine Vorteile bewiesen und zu einer sozialpartnerschaftlichen Krisenbewältigung beigetragen. Trotzdem steigt seit Jahren die Anzahl der in Deutschland ansässigen Unternehmen in ausländischer Rechtsform, die in eine Lücke der Mitbestimmungsgesetze fallen und keinen mitbestimmten Aufsichtsrat bilden. Die hier vorgelegte Untersuchung belegt den steigenden Trend anhand der bis Oktober 2010 bekanntgewordenen Fälle.

\section{Zuzug ausländischer Rechtsformen ohne Mit- bestimmung}

Aufgrund der jüngeren Rechtsprechung des Europäischen Gerichtshofs (EuGH) zur Niederlassungsfreiheit können ausländische Kapitalgesellschaften der Europäischen Union (EU) und des Europäischen Wirtschaftsraums (EWR) sich in der ursprünglichen Rechtsform des Gründungsstaates in Deutschland niederlassen und tätig werden. Konsequenz dessen ist, dass auch deutsche Unternehmer als Rechtsträger für ihre heimischen Aktivitäten eine ausländische Gesellschaft verwenden können. In diesem Fall kommt hierzulande ausländisches Gesellschaftsrecht zur Anwendung. Dies hat Folgen unter anderem für die Mitbestimmung. Betroffen ist die Unternehmensmitbestimmung im Aufsichtsrat, nicht aber die betriebliche Mitbestimmung. Denn für Letztere gilt weiterhin das Betriebsverfassungsgesetz, weil es nicht an das Unternehmen und dessen Rechtsform, sondern an den jeweiligen Betrieb anknüpft.

Nach den Grundsatzentscheidungen des EuGH in Sachen Centros, Überseering und Inspire $\mathrm{Art}^{1}$ ist also ein Zuzug von Gesellschaften möglich, die nach dem Recht eines anderen Mitgliedstaates gegründet wurden. Anders als zuvor müssen solche Unternehmen sich, wenn sie ihren Sitz in Deutschland nehmen wollen, nicht mehr in einer deutschen Rechtsform neu gründen. Die zwar umstrittene, aber herrschende Meinung lehnt in diesen Fällen eine Anwendung der aus der AG und der $\mathrm{GmbH}$ bekannten Unternehmensmitbestimmung auf ausländische Rechtsformen ab, weil die Gesetze nur deutsche Unternehmensformen enumerativ einbeziehen und eine analoge Anwendung nicht in Betracht komme (vgl. Weiss/Seifert 2009, S. 546ff. mit weiteren Nachweisen; Binz/Sorg 2010, $\$ 25, \mathrm{Rn}$. 99ff.; andere Ansicht u.a. Seyboth 2008). Daher müssen diese Unternehmen in der Praxis weder einen Aufsichtsrat bilden, der nach dem Drittelbeteiligungsgesetz zu einem Drittel mit Arbeitnehmervertretern besetzt wäre, wenn sie hierzulande mehr als 500 Arbeitnehmer beschäftigen, noch müssen sie ab 2.000 Arbeitnehmern einen paritätisch besetzten Aufsichtsrat nach dem Mitbestimmungsgesetz von 1976 bilden.

Für US-Unternehmen regelt bereits der Freundschafts-, Handels- und Schifffahrtsvertrag zwischen Deutschland und den Vereinigten Staaten vom 29. Oktober 1954 Entsprechendes (einen Überblick über die bestehenden bilateralen Abkommen gibt Kindler 2006, Rn. 308ff.). Vergleichbares gilt im Verhältnis von Deutschland und der Schweiz aufgrund eines Abkommens über die Freizügigkeit zwischen der Schweizerischen Eidgenossenschaft einerseits und der Europäischen Gemeinschaft und ihren Mitgliedstaaten andererseits.
Die Sorge vor einer groß angelegten „Flucht aus der Mitbestimmung“ hat sich zwar bislang nicht bewahrheitet. Als Massenphänomen wurde die britische Limited nur für Existenzgründer wie kleine Friseuroder Handwerksläden interessant (Empirie bei Niemeier 2007, S. 1974ff.). ${ }^{2}$ Dennoch nutzen immer häufiger auch große Un-

\footnotetext{
1 EuGH vom 9.3.1999, Rs. C-212/97, Slg. 1999, I-1459ff (Centros); EuGH vom 5.11.2002, RS C-208/00, Slg. 2002, I-9919ff (Überseering); EuGH vom 30.9.2003, Rs. C-167/01, NJW 2003 3331 (Inspire Art); ebenso EuGH vom 13.12.2005, Rs. C-411/03, Slg. 2005, I-10805ff (SEVIC Systems $A G$ )

2 Die "Go Ahead Limited Beratung" gibt beispielsweise an, bereits mehr als 39.000 Limiteds gegründet zu haben, http://www.go-limited.de (Stand 25.11.2010). Der Gesetzgeber begegnete der Konkurrenz zur GmbH 2008 mit der Schaffung einer mindestkapitalfreien Unternehmergesellschaft als einfache Variante der $\mathrm{GmbH}$ speziell für Existenzgründer.
}

Sebastian Sick, Dr. jur. und LL.M. Eur., leitet das Referat Wirtschaftsrecht II in der Hans-Böckler-Stiftung. Arbeitsschwerpunkte: Unternehmensrecht, europäisches Gesellschaftsrecht, Unternehmensmitbestimmung, Corporate Governance. e-mail: Sebastian-Sick@boeckler.de Lasse Pütz, Ass. jur., leitet das Referat Wirtschaftsrecht III in der Hans-Böckler-Stiftung im Bereich Mitbestimmungsförderung. Arbeitsschwerpunkte: Unternehmensrecht, Unternehmensmitbestimmung. e-mail: Lasse-Puetz@boeckler.de 
ternehmen die beschriebene Möglichkeit. Prominente Beispiele sind die Fluggesellschaft Air Berlin, die Drogeriemarktkette Müller, die Modekette H\&M oder die Logistikunternehmen Kühne+Nagel sowie Dachser. Für zahlreiche Arbeitnehmer in Deutschland finden deshalb die Mitbestimmungsgesetze keine Anwendung.

Nicht zuletzt durch die Europäisierung sind damit Lücken im Schutzbereich der Unternehmensmitbestimmung entstanden (hierzu auch Thannisch 2010). Angesichts dessen wird immer wieder diskutiert, ob die bundesdeutschen Vorschriften über die unternehmerische Mitbestimmung auf diese sogenannten Scheinauslandsgesellschaften erstreckt werden können. Eine solche Erstreckung forderten die Gewerkschaften schon 2006 in der Regierungskommission zur Modernisierung der deutschen Unternehmensmitbestimmung (Biedenkopfkommission II). Zuletzt im Sommer 2010 wurde diese Forderung durch einen einstimmigen Beschluss des 19. DGBBundeskongresses erneuert (Antrag J 001). ${ }^{3}$

Diese Forderungen sind auch vor dem Hintergrund der Feststellung der wissenschaftlichen Mitglieder der Biedenkopfkommission II (2006, S.73f.; ausführlich Weiss/Seifert, S. 547ff.) zu verstehen, wonach ein Gesetz zur Erstreckung der Mitbestimmung auf ausländische Kapitalgesellschaftsformen grundsätzlich europarechtlich zulässig sei: „Sie [die wissenschaftlichen Mitglieder der Kommission] sind allerdings - in Übereinstimmung mit der wohl überwiegenden Meinung im rechtswissenschaftlichen Schrifttum - überzeugt, dass der deutsche Gesetzgeber gemeinschaftsrechtlich nicht daran gehindert ist, solche Unternehmen jedenfalls dann der Mitbestimmung zu unterwerfen, wenn sich ihre betriebliche Organisation einschließlich der Arbeitnehmer im Wesentlichen im Inland befindet und diese Arbeitnehmer nicht nach dem Recht des Gründungsstaats ein Mitbestimmungsrecht haben."

Die wissenschaftlichen Mitglieder der Kommission sahen allerdings entgegen der gewerkschaftlichen Forderung von einer entsprechenden Empfehlung an den Gesetzgeber ab, weil sie die Zahl der relevanten Fälle zum damaligen Zeitpunkt (Sick 2006) für zu gering erachteten. Sie empfahlen dem Gesetzgeber ,jedoch, die Bildung solcher Unternehmen aufmerksam zu beobachten und in dem Fall, dass sie in nennenswerter Zahl in mitbestimmungsrelevanter Größenordnung auftre- ten, geeignete und gemeinschaftsrechtlich zulässige Maßnahmen zur Aufrechterhaltung der Funktionsfähigkeit der Mitbestimmung zu treffen." (Biedenkopfkommission II 2006, S. 35).

\section{Untersuchung des mit- bestimmungsrelevanten Bereichs}

Die vorliegende Untersuchung nimmt den Ansatz der Biedenkopfkommission II auf, „die Bildung solcher Unternehmen aufmerksam zu beobachten", und analysiert seit nunmehr 2006 regelmäßig Auslandgesellschaften in Deutschland. Die Recherche gilt sämtlichen europäischen Kapitalgesellschaftsformen, wie sie in der SE-Verordnung ${ }^{4}$ aufgelistet sind. Außerdem sind entsprechende US-amerikanische sowie schweizer Rechtsformen erfasst. Als Informationsgrundlagen dienen die Unternehmensdatenbanken von Wiso und Lexis Nexis, insbesondere von Hoppenstedt und Creditreform, aber auch bedirect, D\&B sowie das Handelsregister. Daneben werden zusätzlich Quellen im Internet, der Internetauftritt des jeweiligen Unternehmens sowie Presseartikel genutzt. Sämtliche Daten werden, soweit erhältlich, mit verschiedenen Quellen auf Plausibilität hin abgeglichen. Die Untersuchung erstreckt sich auf Unternehmen, die in den mitbestimmungsrelevanten Bereich fallen, weil sie in Deutschland die für die Drittelbeteiligung relevante Schwelle von 500 Beschäftigen überschreiten. ${ }^{5}$

Die so gewonnenen Daten werden in Abschnitt 3 mit der bereits 2006 im Rahmen der Biedenkopfkommission II vorgelegten und seither erneuerten Empirie verglichen (Sick 2006; 2008; 2010). Daraus ergeben sich Schlussfolgerungen und Handlungsbedarf (Abschnitt 4).

Rechtlich unterscheidet die Untersuchung zwei Formen der Nutzung ausländischer Kapitalgesellschaften:

(1) Einerseits geht es um Kapitalgesellschaften ausländischen Rechts, die in Deutschland direkt mittels unselbstständiger Niederlassung (Zweig- oder Hauptniederlassung bzw. Verwaltungssitz) ${ }^{6}$ tätig sind.

(2) Andererseits - und die derzeitige anwaltliche Beratungspraxis scheint diese
Gestaltungsweise zu bevorzugen - geht es um deutsche Kommanditgesellschaften, die als persönlich haftende Gesellschafter (Komplementär) eine ausländische Rechtsform nutzen (z. B. Ltd. \& Co. KG, zu dieser Rechtsform ausführlich Werner 2006). Diese Möglichkeit, die früher schon vereinzelt versucht wurde, war zwar seit jeher auch bei den Gerichten umstritten (Werner 2006; Kisker 2008, S. 76f.), sie hat aber durch die europäische Rechtsprechung zur Niederlassungsfreiheit neue Beachtung und Bestätigung gefunden. Beschäftigt die ausländische Kapitalgesellschaft \& Co. KG mehr als 2.000 Arbeitnehmer, kann die Mitbestimmung auf diese Weise in der Praxis umgangen werden. Der Wortlaut des Mitbestimmungsgesetzes $(\$ 4, \$ 1$ MitbestG) erfasst direkt nur die deutsche Kapitalgesellschaft \& Co. KG (z. B. GmbH \& Co. KG). Und vom Drittelbeteiligungsgesetz wird die Kapitalgesellschaft \& Co. KG - entgegen der Empfehlung der wissenschaftlichen Mitglieder der Biedenkopfkommission II - überhaupt nicht erfasst, weder die deutsche noch die ausländische.

\section{Die Lücke wächst}

Seit 2006 sind 26 mitbestimmungsrelevante Fälle der Nutzung ausländischer Rechtsformen hinzugekommen. Diese Steigerung

3 Vgl. auch die entsprechenden Anträge der SPDFraktion (BT-Dr. 17/2122 vom 16.6.2010) und der Fraktion DIE LINKE (BT-Dr. 17/1413 vom 21.4.2010) im Deutschen Bundestag.

4 Gemäß Art. 2 Abs. 1 und 2 in Verbindung mit Anhang I und II der VO Nr. 2157/2001/EG vom 8.10.2001.

5 Die Abgrenzung erfolgt nach den gesetzlichen Schwellenwerten (500/2000). Weitere Voraussetzungen des DrittelbG ( $\S 2$ ) oder des MitbestG $(\S \S 4,5)$ sind nicht Gegenstand der Untersuchung. Weil die Datenbanken nicht durchgängig zwischen Unternehmens- und Konzernmitarbeitern unterscheiden, sind leichte Abweichungen in Einzelfällen nicht gänzlich auszuschließen.

6 Betrachtet werden Gesellschaften mit Handelsregistereintrag in Deutschland, wie nach §§13d ff. $\mathrm{HGB}$ vorgeschrieben. Das Eintragungserfordernis für Zweigniederlassungen gem. §§13d ff. HGB gilt nach herrschender Meinung auch für die von der Rechtsprechung des EuGH zur Niederlassungsfreiheit behandelten ausländischen Gesellschaften mit Satzungssitz in der EU, aber Verwaltungssitz (Hauptniederlassung) in Deutschland. Die Konsequenzen für die Mitbestimmung sind in den Fällen der "gewöhnlichen" Zweigniederlassung und des Verwaltungssitzes identisch, weshalb hier eine Unterscheidung nicht erfolgt. 
Tabelle 1: Unternehmen, die der deutschen Mitbestimmung entzogen sind

\begin{tabular}{|c|c|c|c|c|c|c|c|c|}
\hline \multirow[b]{2}{*}{$\begin{array}{l}\text { Arbeitnehmer in } \\
\text { Deutschland }\end{array}$} & \multicolumn{2}{|c|}{$\begin{array}{c}\text { Deutsche Personengesellschaft } \\
\text { mit ausländischem } \\
\text { Komplementär }\end{array}$} & \multicolumn{2}{|c|}{$\begin{array}{c}\text { Ausländische } \\
\text { Kapitalgesellschaft mit Nieder- } \\
\text { lassung in Deutschland }\end{array}$} & \multicolumn{2}{|c|}{ Gesamt } & \multicolumn{2}{|c|}{$\begin{array}{l}\text { Konzerne mit einem oder } \\
\text { mehreren betroffenen } \\
\text { Unternehmen }\end{array}$} \\
\hline & $>500$ & $>2000$ & $>500$ & $>2000$ & $>500$ & $>2000$ & $>500$ & $>2000$ \\
\hline Jan. 2006 & 11 & 9 & 6 & 1 & 17 & 10 & 17 & 10 \\
\hline Jan. 2008 & 19 & 15 & 10 & 1 & 29 & 16 & 28 & 15 \\
\hline Nov. 2009 & 21 & 14 & 16 & 2 & 37 & 16 & 35 & 16 \\
\hline Okt. 2010 & 27 & 17 & 16 & 2 & 43 & 19 & 39 & 16 \\
\hline Zunahme seit 2006 & 16 & 8 & 10 & 1 & 26 & 9 & 22 & 6 \\
\hline Beispiele & \multicolumn{2}{|c|}{$\begin{array}{l}\text { Müller Ltd.\&Co.KG } \\
\text { H\&M B.V.\&Co.KG } \\
\text { Air Berlin Plc\&Co.KG }\end{array}$} & \multicolumn{2}{|c|}{$\begin{array}{l}\text { McDonlads's Deutschland Inc. } \\
\text { McKinsey\&Comp. Inc. } \\
\text { Cortal Consors S.A. }\end{array}$} & & & \multicolumn{2}{|c|}{$\begin{array}{l}\text { Air Berlin } \\
\text { H\&M } \\
\text { QVC }\end{array}$} \\
\hline
\end{tabular}

auf insgesamt 43 Unternehmen bedeutet weit mehr als eine Verdoppelung innerhalb von weniger als fünf Jahren. Allein im letzten Jahr ist die Zahl der Gesellschaften um sechs gewachsen. Die Oracle GmbH hat sich beispielsweise erst mit Gesellschafterbeschluss vom 31.5.2010 in die Oracle Deutschland B.V. \& Co. KG umgewandelt. Die 43 Unternehmen mit mehr als 500 Arbeitnehmern in Deutschland verteilen sich auf nunmehr 39 Konzerne, in denen mindestens eine Gesellschaft betroffen ist (Tabelle 1 und Abbildung 1). In drei Konzernen existieren bereits Zweit- oder Drittgründungen von Gesellschaften mit ausländischer Rechtsform, die jeweils mit ihren Beschäftigtenzahlen in den mitbestimmungsrelevanten Bereich fallen. Nachdem 2006 noch kein solcher Fall bekannt war, sind hier die Fluggesellschaft Air Berlin mit den Gesellschaften Air Berlin PLC \& Co. Luftverkehrs KG und Air Berlin PLC \& Co. Cabin Service KG sowie die Bekleidungskette H\&M mit ihren Gesellschaften H\&M Hennes \& Mauritz B.V. \& Co. KG und H\&M Hennes \& Mauritz Logistik AB \& Co. KG sowie die QVC Gruppe mit den Gesellschaften QVC Deutschland Inc. \& Co. KG, QVC eDistribution Inc. \& Co. KG und QVC eServices Inc. \& Co. KG zu nennen.

Beschränkt man die Betrachtung auf die Unternehmen, die aufgrund der ausländischen Rechtsform aus dem Mitbestimmungsgesetz von 1976 herausfallen, die also die Schwelle von 2.000 Arbeitnehmern überschreiten, so zeigt sich immer noch ein Anstieg von zehn (2006) auf 19 (2010) Gesellschaften (Tabelle 1, Übersichten 1 und 2).

\section{Abb. 1: Unternehmen mit ausländischer Rechtsform und mehr als 500 Arbeitnehmern in Deutschland}

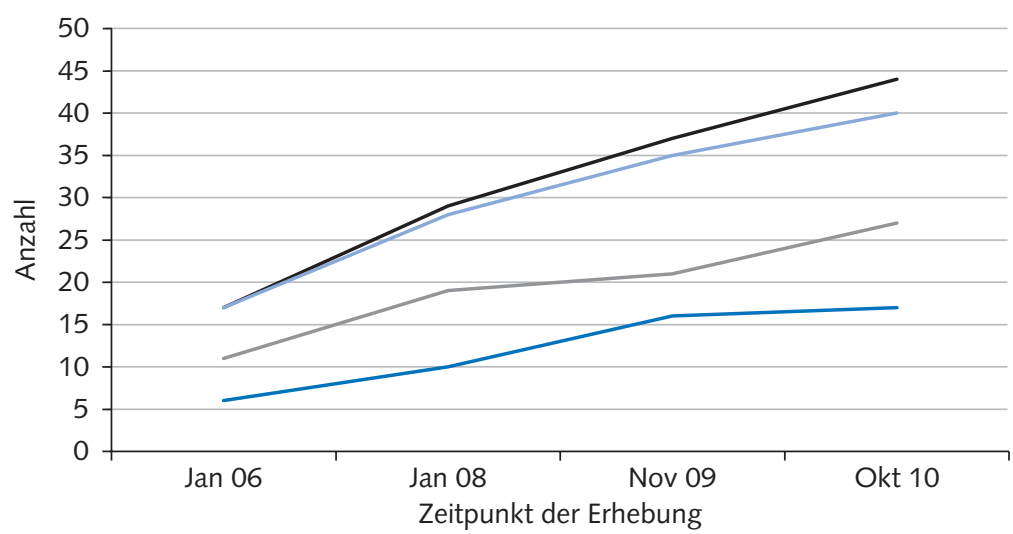

\subsection{PERSONENGESELLSCHAFTEN MIT AUSLÄNDISCHEM KOMPLE- MENTÄR UND NIEDERLASSUNGEN AUSLÄNDISCHER UNTERNEHMEN}

Die 43 bekannten Fällekönnen weiter unterschieden werden nach Personengesellschaften mit ausländischer Kapitalgesellschaft als persönlich haftendem Gesellschafter (Komplementär) und nach ausländischen Kapitalgesellschaften mit Niederlassung in Deutschland. ${ }^{7}$ Die erste Rubrik bildet mit 27 Unternehmen, von denen 16 über der Schwelle von 2.000 Arbeitnehmern liegen, den größeren Bereich. 2006 waren es noch lediglich elf Gesellschaften. Die Zahl der ausländischen Kapitalgesellschaften, die mit unselbstständigen Niederlassungen (Hauptniederlassung bzw. Verwaltungssitz oder Zweigniederlassung) in den mitbe-

\footnotetext{
Sofern ausländische Komplementärgesellschaften von Personengesellschaften zugleich mit eigenem Verwaltungssitz in Deutschland auftreten, so wurden sie ausschließlich in der Gruppe der Personengesellschaft mit ausländischem Komplementär aufgenommen, nicht aber zusätzlich bei den Niederlassungen ausländischer Gesellschaften. So wurden Doppelzählungen vermieden (z.B. Air Berlin PLC mit Niederlassung in Deutschland als Komplementär in der Air Berlin PLC \& Co. Luftverkehrs KG).
} 


\section{Übersicht 1: Personengesellschaften mit ausländischer Kapitalgesellschaft als Komplementär und mit mehr als 500 Beschäftigten in Deutschland}

\begin{tabular}{|c|c|c|c|}
\hline & Firmenname & Geschäftsfeld & Beschäftigte (D) \\
\hline 1 & Müller Ltd. \& Co. KG & Einzelhandel mit Drogerieartikeln & $12.001-20.000$ \\
\hline 2 & United Parcel Service Deutschland Inc. \& Co. OHG & Paket- und Kurierdienst & $12.001-20.000$ \\
\hline 3 & Gegenbauer Holding SA \& Co. KG & Gebäudereinigung und Managementtätigkeiten & $10.001-12.000$ \\
\hline 4 & Air Berlin PLC \& Co. Luftverkehrs KG & Fluggesellschaft & $5.001-10.000$ \\
\hline 5 & Dachser GmbH (Österreich) \& Co.KG & Logistik- und Gütertransportunternehmen & $5.001-10.000$ \\
\hline 6 & H\&M Hennes \& Mauritz B.V. \& Co. KG & Bekleidungshandel & $5.001-10.000$ \\
\hline 7 & K \& K Klaas \& Kock B.V. + Co. KG & Einzelhandel mit Lebensmittel und Non-Food-Artikel & $5.001-10.000$ \\
\hline 8 & Kühne + Nagel AG (Luxemburg) \& Co. KG & Logistik- und Gütertransportunternehmen & $5.001-10.000$ \\
\hline 9 & Sodexo Beteiligungs B.V. \& Co. KG & Catering und Facility Services & $5.001-10.000$ \\
\hline 10 & Apetito catering B.V. \& Co. KG & Bewirtschaftung von Einrichtungen der Gemeinschaftsverpflegung & $2.001-5.000$ \\
\hline 11 & Autoliv B.V. \& Co. KG & Hersteller von Zubehör für Kraftwagen & $2.001-5.000$ \\
\hline 12 & DIW Instandhaltung Ltd. \& Co. KG. & Gebäudereinigung & $2.001-5.000$ \\
\hline 13 & Esprit Retail B.V. \& Co. KG & Bekleidungshandel & $2.001-5.000$ \\
\hline 14 & Huhtamaki Deutschland GmbH \& Co. KG & Verpackungsproduzent für Lebensmittel- und Non-Food-Bereich & $2.001-5.000$ \\
\hline 15 & Prinovis Ltd. \& Co. KG & Druckereiunternehmen & $2.001-5.000$ \\
\hline 16 & QVC Deutschland Inc. \& Co. KG & Holding im Bereich Teleshopping & $2.001-5.000$ \\
\hline 17 & Rolls-Royce Deutschland Ltd. \& Co. KG & $\begin{array}{l}\text { Luft- und Raumfahrzeugbau, Herstellung von Verbrennungsmotoren und } \\
\text { Turbinen }\end{array}$ & $2.001-5.000$ \\
\hline 18 & Air Berlin PLC \& Co. Cabin Service KG & $\begin{array}{l}\text { Dienstleistungen für Luftverkehrsunternehmen sowie die Überlassung von } \\
\text { Flugbegleitern, Bodenpersonal und Piloten }\end{array}$ & $501-2.000$ \\
\hline 19 & Boeing International B.V. \& Co. Holding KGaA & Flugzeugbau und Vertrieb & $501-2.000$ \\
\hline 20 & EUROPCAR INTERNATIONAL S.A. \& CO. OHG & Autovermietung & $501-2.000$ \\
\hline 21 & $\begin{array}{l}\text { GLOBALFOUNDRIES Dresden Module One } \\
\text { Limited Liability Company \& Co. KG. }\end{array}$ & Herstellung und Vertrieb von Mikroprozessoren & $501-2.000$ \\
\hline 22 & H\&M Hennes \& Mauritz Logistik A.B. \& Co. KG & Herstellung, Vertrieb und Logistik sowie Im- und Export von Waren & $501-2.000$ \\
\hline 23 & Maersk Deutschland A/S \& Co. KG & Reedereivertretungen, Befrachtungen usw. & $501-2.000$ \\
\hline 24 & ORACLE Deutschland B.V. \& Co. KG & Anbieter von universellen Informations-Management-Lösungen & $501-2.000$ \\
\hline 25 & QVC eDistribution Inc. \& Co. KG & Distribution von Waren aller Art & $501-2.000$ \\
\hline 26 & QVC eServices Inc. \& Co. KG & Call-Center & $501-2.000$ \\
\hline 27 & Sykes Enterprises Support Services B.V. \& Co. KG & Call-Center & $501-2.000$ \\
\hline
\end{tabular}

\section{Übersicht 2: Niederlassungen ausländischer Unternehmen in Deutschland mit mehr als 500 Beschäftigten in Deutschland}

\begin{tabular}{|c|c|c|c|}
\hline & Firmenname & Geschäftsfeld & Beschäftigte (D) \\
\hline 1 & McDonald's Deutschland Inc. & Gastronomie & $10.001-12.000$ \\
\hline 2 & Deere \& Company, Inc. & Großhandel mit und Produktion von Landmaschinen und Geräten & $5.001-10.000$ \\
\hline 3 & AIDA Cruises - German Branch of Costa Crociere S.p.A. & Reiseveranstalter und Reederei & $501-2.000$ \\
\hline 4 & American Express International Inc. & Finanz- und reisebezogene Dienstleistung & $501-2.000$ \\
\hline 5 & $\begin{array}{l}\text { Atradius Kreditversicherung, Niederlassung der Atradius Credit } \\
\text { Insurance N.V. }\end{array}$ & Kreditversicherer & $501-2.000$ \\
\hline 6 & BNP Paribas Personal Finance S.A. & Kreditbank & $501-2.000$ \\
\hline 7 & Cortal Consors S.A. & Bankgeschäfte & $501-2.000$ \\
\hline 8 & CWT Beheermaatschappij B.V. Deutschland & Reisebürokette im Geschäftsreisesektor & $501-2.000$ \\
\hline 9 & Federal Express Europe Inc. & Paket- und Kurierdienst & $501-2.000$ \\
\hline 10 & Ford Bank Niederlassung des FCE Bank PLC & Automobilbank & $501-2.000$ \\
\hline 11 & Helvetia Schweizerische Versicherungsgesellschaft AG (Schweiz) & Schaden- und Unfallversicherung & $501-2.000$ \\
\hline 12 & McKinsey \& Company, Inc. & Beratung auf dem Gebiet der Unternehmensführung & $501-2.000$ \\
\hline 13 & Mitsubishi Electric Europe B.V. & $\begin{array}{l}\text { Großhandel mit elektrischen Haushaltsgeräten und Geräten der } \\
\text { Unterhaltungselektronik }\end{array}$ & $501-2.000$ \\
\hline 14 & $\begin{array}{l}\text { RCI (Renault Credit International) Banque S.A. Niederlassung } \\
\text { Deutschland }\end{array}$ & Automobilbank & $501-2.000$ \\
\hline 15 & Swiss Life AG (Schweiz) & Versicherer & $501-2.000$ \\
\hline 16 & Weleda AG (Schweiz) Schwäbisch Gmünd & $\begin{array}{l}\text { Entwicklung, Herstellung und Vertrieb von pharmazeutischen und } \\
\text { kosmetischen Präparaten }\end{array}$ & $501-2.000$ \\
\hline
\end{tabular}


stimmungsrelevanten Bereich fallen, nahm im selben Zeitraum von sechs auf 16 Unternehmen zu und hat sich somit beinahe verdreifacht (Tabelle 1, 2 und 3).

\subsection{BRANCHENAUFFÄLLIGKEITEN}

Ein großer Anteil der 43 Unternehmen stammt aus den Branchen Verkehr und Nachrichtenübermittlung ${ }^{8}$ (neun Unternehmen), Kredit- und Versicherungsgewerbe $^{9}$ (acht Unternehmen) sowie Einzelhandel $^{10}$ (fünf Unternehmen). ${ }^{11}$ Die übrigen Gesellschaften verteilen sich auf unterschiedliche Branchen. Die acht Unternehmen des Kredit- und Versicherungsgewerbes haben alle zwischen 500 und 2.000 Arbeitnehmer; sie treten ausschließlich in der Form der Niederlassung auf und bilden so etwa die Hälfte der ermittelten Fälle in dieser Rubrik. Alle Unternehmen der Einzelhandelsbranche haben demgegenüber mehr als 2.000 Arbeitnehmer und treten ausschließlich als ausländische $\mathrm{Ka}$ pitalgesellschaften \& Co. KG auf.

\subsection{ZUNAHME EUROPÄISCHER RECHTSFORMEN}

Des Weiteren verdeutlicht die Untersuchung eine relative Zunahme der Nutzung europäischer Rechtsformen (33) gegenüber solchen der USA (10) (Tabelle 2 und 3). ${ }^{12}$ Dieser Befund macht die praktische Auswirkung der eingangs zitierten europäischen Rechtsprechung zusätzlich augenscheinlich. Bemerkenswert ist, dass entgegen der landläufigen Einschätzung nicht die britische Limited am häufigsten genutzt wurde, sondern die niederländischen Gesellschaftsmodelle (11 B.V., 1 N.V.). Darauf folgen die amerikanischen Formen (10 Inc./LLC) und erst dann die britische Limited (7 Ltd./PLC). Bei den Personengesellschaften mit ausländischem Komplementär überwiegen ebenfalls die europäischen Rechtsformen: Die niederländische B.V. mit neun und die britische Limited mit sechs Gesellschaften, wogegen nur fünfmal eine US-amerikanische Gesellschaftsform vertreten ist. Anders als noch 2006 überwiegen auch bei den Niederlassungen die europäischen Rechtformen mit elf Unternehmen (2006: 2) gegenüber fünf US-amerikanischen Rechtsformen (2006: 4). Unter den Niederlassungen ausländischer Gesellschaften hat sich der Anteil US-amerikanischer Unternehmen demzufolge seit 2006 von ca.

Tabelle 2: Personengesellschaft mit ausländischer Kapitalgesellschaft als Komplementär

\begin{tabular}{|c|c|c|c|c|}
\hline & $\begin{array}{c}\text { Januar } \\
2006\end{array}$ & $\begin{array}{c}\text { Januar } \\
2008\end{array}$ & $\begin{array}{c}\text { November } \\
2009\end{array}$ & $\begin{array}{c}\text { Oktober } \\
2010\end{array}$ \\
\hline Gesamt (über $500 \mathrm{AN}$ in D) & 11 & 19 & 21 & 27 \\
\hline Über 2000 AN in D & 9 & 15 & 14 & 17 \\
\hline Komlementär in US-amerikanischer Rechtsform & 2 & 4 & 4 & 5 \\
\hline Komplementär in europäischer Rechtsform & 9 & 15 & 17 & 22 \\
\hline
\end{tabular}

Tabelle 3: Ausländische Kapitalgesellschaft mit Niederlassung in Deutschland

\begin{tabular}{lcccc}
\hline & $\begin{array}{c}\text { Januar } \\
\text { 2006 }\end{array}$ & $\begin{array}{c}\text { Januar } \\
\mathbf{2 0 0 8}\end{array}$ & $\begin{array}{c}\text { November } \\
\mathbf{2 0 0 9}\end{array}$ & $\begin{array}{c}\text { Oktober } \\
\mathbf{2 0 1 0}\end{array}$ \\
\hline Gesamt (über 500 AN in D) & 6 & 10 & 16 & 16 \\
Mehr als 2000 AN in D & 1 & 1 & 2 & 2 \\
Aus USA & 4 & 4 & 5 & 5 \\
Europ. Rechtsform & 2 & 6 & 11 & 11 \\
\hline & & & WSI MITEILUNGEN \\
Quelle: Erhebung der Autoren. & & & MS
\end{tabular}

$67 \%$ auf rund $31 \%$ umgekehrt. Von den elf europäischen Gesellschaftsformen sind acht solche des EWR, drei sind schweizer Aktiengesellschaften.

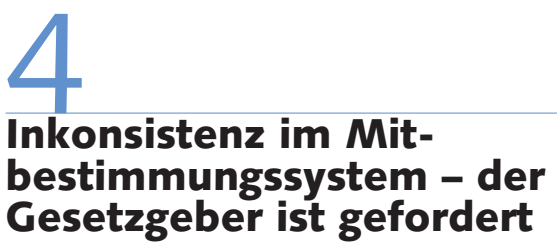

Die vorliegende Untersuchung verdeutlicht zunächst, dass weiterhin das von Mitbestimmungskritikern zuweilen verbreitete Bild einer Sturmflut von Auslandsgesellschaften, die unter Vermeidung der Mitbestimmung in Deutschland tätig werden, nicht mit der Realität übereinstimmt. Angesichts von 682 (quasi-)paritätisch mitbestimmten (Stand 31.12.2010; Ehrenstein 2010) und ca. 1.500 drittelbeteiligten Unternehmen (Stand 31.12.2009; Bayer 2009) lässt die Zahl von 43 Unternehmen ausländischer Rechtsform nicht auf eine verbreitete Flucht schließen, die die deutsche Mitbestimmung infrage stellen könnte. Diesbezüglich weist auch die Faktenlage zur Europäischen Aktiengesellschaft (SE) nicht in eine andere Richtung. Bis November 2010 waren lediglich 28 SE mit mehr als 500 Arbeitnehmern in Deutschland registriert (Köstler 2010). Demzufolge besteht kein sachlich begründeter Handlungsdruck dahingehend, das Niveau oder die
Verbindlichkeit der gesetzlichen Mitbestimmung in Deutschland aufzuweichen, um eine zuweilen behauptete Erosion des Systems zu vermeiden. Auch ein „Austrocknen“ der AG oder GmbH aufgrund

8 Klassifikation der Wirtschaftszweige 2003 (WZ 2003), Ziff. 60-64.

9 WZ 2003, Ziff. 65-66.

10 WZ 2003, Ziff. 52

11 Über die Umwandlung des deutschen Zweigs der Modekette ZARA in eine B.V. \& Co. KG, bei der die Arbeitnehmer beschäftigt werden, wurde zwar bereits in der Presse berichtet. Der abschließende Vollzug dieser Ankündigung konnte erst nach Abschluss der Studie im Oktober 2010 der Bekanntmachung im Handelsregister vom 2.11.2010 definitiv entnommen werden.

12 Folgende Rechtsformen sind aufgetreten (vgl. dazu Wachter 2006): A.B. = Aktiebolag, Aktiengesellschaft nach schwedischem Recht; AC = Aktiengesellschaft nach schweizer Recht / luxemburgischem Recht; $A / S=$ Aktieselskab, Aktiengesellschaft nach dänischem Recht; B.V. = Besloten Vennootschap, Kapitalgesellschaft niederländischen Rechts, ähnlich der $\mathrm{GmbH} ; \mathrm{GmbH}$ / Ges.mbh = Gesellschaft mit beschränkter Haftung österreichischen Rechts; Inc. = Incorporated Company, US-amerikanische Rechtsform; LLC = Limited Liability Company, US-amerikanische Rechtsform; Ltd. / LTD = Private Limited Company By Shares, britische Rechtsform, ähnlich der $\mathrm{GmbH}$; N.V. = Naamloze Vennootschap, die Bezeichnung für eine Aktiengesellschaft nach niederländischem Recht; PLC = Public Company Limited By Shares, britische Rechtsform, ähnlich der Aktiengesellschaft; S.A. = Société anonyme, Aktiengesellschaft französischen / luxemburgischen Rechts; S.p.A = Societa per Azioni; Aktiengesellschaft nach italienischen Recht. 
der Mitbestimmung ist offenbar nicht zu befürchten (so auch Köstler 2009; andere Ansicht Arbeitskreis Unternehmerische Mitbestimmung 2009 sowie Teichmann 2009).

Gleichwohl kann keine Entwarnung für die deutsche Mitbestimmung gegeben werden. Denn die vorliegende Studie zeigt zugleich, dass die Zahl der mitbestimmungsrelevanten Fälle seit Abschluss der Biedenkopfkommission II deutlich ansteigt. Durch die eingangs zitierte Rechtsprechung des EuGH zur Niederlassungsfreiheit hat sich offenbar eine Lücke in den Gesetzen aufgetan, die zu Inkonsistenzen im deutschen Mitbestimmungssystem führt. Von Zufälligkeiten kann angesichts dieser Steigerung nicht mehr die Rede sein. Schließlich bietet sich die Scheinauslandsgesellschaft der anwaltlichen Beratungspraxis als eine Option im Strauß der Möglichkeiten zur Vermeidung der Mitbestimmung an. Die Konstruktion mit ausländischer Rechtsform wird von ausländischen Konzernen wie Rolls Royce oder UPS genauso wie von großen deutschen Familienunternehmen wie der Drogeriemarktkette Müller genutzt. Auffällig ist aber die Häufung im Einzelhandel und in der Logistiksparte (Verkehr- und Nachrichtenübermittlung) sowie im Kredit- und Versicherungsgewerbe.

Firmen, die eine ausländische Rechtsform führen, suggerieren als Grund zuweilen eine einfachere Koordination ihrer internationalen Tätigkeiten oder propagieren die Limited \& Co. KG als vermeintlich kostengünstigere Alternative zur $\mathrm{GmbH}$. Es häufen sich aber Fälle, in denen Unternehmen durch den Wechsel der Rechtsform auch gezielt die Unternehmensmitbestimmung vermeiden wollen (vgl.
Werner 2006, S. 41). Dieses Motiv leitete beispielsweise nach eigenen Aussagen Unternehmenschef Joachim Hunold bei der Fluggesellschaft Air Berlin, die als PLC \& Co. KG firmiert (Süddeutsche Zeitung vom 31.3.2010, S. 22). Auch die deutsche Tochter des schwedischen Textilhändlers $\mathrm{H} \& \mathrm{M}$ wechselte von der $\mathrm{GmbH}$ in eine B.V. \& Co. KG gerade zu dem Zeitpunkt, als die Betriebsräte einen mitbestimmten Aufsichtsrat durchsetzen wollten. Ähnlich liegen die Fälle der Modekette Esprit und der Großspedition Kühne+Nagel. Konzerne wie Air Berlin und H\&M gehen nunmehr schon zur Gründung von Zweit- oder Drittauslandsgesellschaften jeweils dort über, wo eine mitbestimmungsrelevante Größe gegeben ist.

Welche Motive auch immer hinter der Wahl der Unternehmensform stehen, für die Beschäftigten bedeutet der rechtliche Sonderstatus weniger Partizipationsrechte. In der Praxis fallen diese Unternehmen in ausländischen Rechtsformen aus dem deutschen Mitbestimmungsgesetz von 1976 sowie aus dem Drittelbeteiligungsgesetz heraus. Während in einer deutschen Aktiengesellschaft oder $\mathrm{GmbH}$ mit mehr als 2.000 Mitarbeitern die Arbeitnehmer die Hälfte der Aufsichtsräte stellen und bei mehr als 500 bis 2.000 Mitarbeitern der Aufsichtsrat zu einem Drittel aus Arbeitnehmervertretern besteht, haben sie beispielsweise in einer „deutschen“ Limited keinen Anspruch auf Repräsentanz. Dies führt zu einer Benachteiligung eines Teils der Arbeitnehmer in Deutschland. Ein konsistentes Mitbestimmungsrecht müsste jedoch alle Unternehmen erfassen, die ihren organisatorischen Mittelpunkt in Deutschland haben (vgl. Teichmann 2009).
Die Zunahme von 17 auf 43 Fälle in mitbestimmungsrelevanter Größenordnung in nicht einmal fünf Jahren stützt daher die Forderung, durch eine Erstreckung der Mitbestimmungsgesetze auf Auslandsgesellschaften Rechtssicherheit herzustellen, die rechtliche Lücke zu schließen und das Mitbestimmungssystem in dieser Hinsicht europatauglich zu machen. In Betracht käme derzeit nur ein bundesdeutsches Gesetz. Die ebenfalls erwägenswerte Lösung, über eine europäische Sitzverlegungsrichtlinie einen europäischen Schutz der Mitbestimmung zu schaffen und zugleich weitere rechtliche Probleme des „Nomadentums“ von Kapitalgesellschaften einheitlich zu lösen, ist aktuell nicht realistisch. Ein Gesetz europarechtlich zulässig auszugestalten, erscheint aber durchaus möglich, wie bereits Weiss und Seifert (2009) überzeugend dargelegt haben. Die Erhaltung einer mitgliedstaatlichen Mitbestimmungsordnung dürfte als zwingender Grund des Allgemeininteresses insofern auch eine Einschränkung der Niederlassungsfreiheit von Scheinauslandsgesellschaften erlauben. Ein widerspruchsfreies Gesetz müsste Auslandsgesellschaften mit Verwaltungssitz in Deutschland sowie Personengesellschaften mit ausländischem Komplementär erfassen - und das sowohl im Mitbestimmungsgesetz als auch im Drittelbeteiligungsgesetz.

Die deutsche Mitbestimmung hat sich gerade in der Krise bewährt. Sinnvoll erscheint es daher, nicht die Mitbestimmung aufzuweichen, sondern in Europa für generelle Standards der Mitbestimmung einzutreten und national das geltende Mitbestimmungssystem zugleich zu schützen und konsistent anzuwenden. 
Arbeitskreis „Unternehmerische Mitbestimmung“ (2009): Entwurf einer Regelung zur Mitbestimmungsvereinbarung sowie zur Größe des mitbestimmten Aufsichtsrats, in: ZIP - Zeitschrift für Wirtschaftsrecht 19, S. $885-889$

Bayer, W. (2009): Drittelbeteiligung in Deutschland - Ermittlung von Gesellschaften, die dem DrittelbG unterliegen, Untersuchung im Auftrag der Hans-Böckler-Stiftung, http://www.boeckler.de/pdf/mbf drittelbeteiligung.pdf

Binz, M. K./Sorg, M. H. (2010): Die GmbH \& Co. KG, München Ehrenstein, I. (2010): Aktuell 682 mitbestimmte Unternehmen, in: Die Mitbestimmung 6, S. 67

Kindler, P. (2006): Internationales Handels- und Gesellschaftsrecht, Münchener Kommentar zum BGB, München

Kommission zur Modernisierung der deutschen Unternehmensmitbestimmung (Biedenkopfkommission II) (2006): Bericht der wissenschaftlichen Mitglieder der Kommission mit Stellungnahmen der Vertreter der Unternehmen und der Vertreter der Arbeitnehmer, o. O., http://www.bundesregierung.de/Content/DE/Archiv16/Artikel/2006/12/Anlagen/2006-12-20mitbestimmungskommission, property=publicationFile.pdf

Kisker, O. (2006): Unternehmensmitbestimmung bei Auslandsgesellschaften mit Verwaltungssitz in Deutschland, Köln

Köstler, R. (2009): Das trojanische Pferd der verhandelten Mitbestimmung, in: Der Aufsichtsrat 10, S. 137

Köstler, R. (2010): Zur Faktenlage bei der SE, Düsseldorf, http://www. boeckler.de/pdf/bb_mitbestimmung_se_2010_09.pdf.

Niemeier, W. (2007): Die "Mini-GmbH“ (UG) trotz Marktwende bei der Limited, in: ZIP - Zeitschrift für Wirtschaftsrecht 38, S. 1974-1801
Seyboth, M. (2008): Die Mitbestimmung im Lichte der beabsichtigten Neuregelung des Internationalen Gesellschaftsrechts, in: Arbeit und Recht 56 (4), S. 132-136

Sick, S. (2006): Mitbestimmungsrelevante Unternehmen mit ausländischen / kombiniert ausländischen Rechtsformen, in: Biedenkopfkommission II, Anhang 5 = Düsseldorf, http://www.boeckler.de/pdf/ impuls_2006_02_sick.pdf

Sick, S. (2008): Mitbestimmungsrelevante Unternehmen mit ausländischen / kombiniert ausländischen Rechtsformen, Düsseldorf, http:// www.boeckler.de/pdf/mbf_2008_06_19_sick.pdf.

Sick, S. (2010): Mitbestimmungsrelevante Unternehmen mit ausländischen / kombiniert ausländischen Rechtsformen, Datenverz. Nov. 2009, Düsseldorf, http://www.boeckler.de/pdf/mbf_2010_01_20_sick.pdf Teichmann, C. (2009): Verhandelte Mitbestimmung für Auslandsgesellschaften, in: ZIP - Zeitschrift für Wirtschaftsrecht 38, S. 1787-1788 Thannisch, R. (2010): Die Mitbestimmung im Kontext europäischer Herausforderungen, in: WISO direkt, Friedrich-Ebert-Stiftung, Bonn, http://www.fes.de/wiso/pdf/abp/2010/wisodirekt_thannisch.pdf. Wachter, T. (2006): Synoptischer Überblick über die GmbH und EURechtsformalternativen, in: GmbHRundschau Sonderheft September, S. $75-87$

Weiss, M./Seifert, A. (2009): Der europarechtliche Rahmen für ein Mitbestimmungserstreckungsgesetz, in: ZGR - Zeitschrift für Unternehmens- und Gesellschaftsrecht 38 (4), S. 542-580

Werner, R. (2006): Die Ltd. \& Co. KG, GmbHRundschau, Sonderheft September, S. 41-47 